\title{
A Bioestatística em contexto para o estudante de graduação: relato de experiência de uma prática interdisciplinar para o curso de Ciências Biológicas
}

\section{Biostatistics in context for the undergraduate student: experience report of an interdisciplinary practice for the Biological Sciences graduation course}

\author{
Ronaldo Figueiró $^{1}$ | ronaldofigueiro@gmail.com \\ André Barbosa Vargas ${ }^{1}$ | andrebvargas@yahoo.com.br \\ Valéria Vieira $^{1}$ | valvibr@yahoo.com.br
}

\section{RESUMO}

Neste relato de experiência buscou-se ressaltar a necessidade de integrar conhecimentos de diferentes áreas com o objetivo de construir um conhecimento palpável em experiências reais, contextualizadas, com significado para o aprendiz. A experiência pessoal relatada é o resultado de uma atividade prática interdisciplinar que envolveu as disciplinas de Bioestatística, Ecologia e Zoologia. Foi realizada uma prática integrada em campo, com as referidas disciplinas, tendo como uma das finalidades, além de trabalhar a interdisciplinaridade, proporcionar aos alunos a compreensão da importância de Bioestatística em suas futuras atividades como biólogos. Os alunos receberam um projeto de grupo, envolvendo coleta e análise de dados, que foi monitorado periodicamente. A turma, composta por 24 alunos, foi dividida em quatro grupos de seis alunos, sendo cada grupo responsável por seu próprio conjunto de dados, recolhidos por eles mesmos nos jardins do campus universitário. Assim, para cada grupo foi atribuída a tarefa de fazer um relatório de pesquisa, onde deveriam apresentar sua hipótese, analisá-la e comprová-la, explicando os padrões encontrados com base na teoria ecológica. Desta forma, observou-se a construção do raciocínio para testar diferentes hipóteses, iniciado desde a análise dos transectos individuais, até a análise comparativa dessas faixas de observação em relação ao seu posicionamento no campus. Sendo possível constatar que o diálogo entre as disciplinas envolvidas neste estudo, exaltado pelos discentes, proporcionaram um aprendizado prazeroso e contextualizado.

Palavras-chave: Bioestatística, ensino de ciências, interdisciplinaridade. 


\section{ABSTRACT}

In this experience report, the necessity of integrating knowledge from different areas aiming the construction of a palpable knowledge in real, contextualized, meaningful experiences to the apprentice is emphasized. The personal experience reported is the result of a practical interdisciplinary activity involving the disciplines of Biostatistics, Ecology and Zoology. A practical integrated field activity was conducted involving the three disciplines, aiming to, besides working with interdisciplinarity, allow the students the comprehension of the importance of biostatistics in their future activities as biologists. The students received a group Project, involving data collection and analysis, which was periodically monitored. The class, composed of 24 students, was divided in four groups comprised of six students, and each group was responsible for its own data set, sampled by themselves in the gardens of the university campus. Thus, for each group the task of developing a research report, in the format of a scientific manuscript was assigned, where they should present their hyphoteses, analyze and test them, expaining the patterns they observed based in ecological theory. This way, it was observed the construction of thought yo test the different hypotheses, started since the data of individual transects until the comparative analysis of these observation lines in relation to their geographical position within the campus. It was possible to verify that the dialogue among the disciplines involved in this activity, exalted by the students, allowed a pleasant and contextualized learning.

Keywords: Biostatistics, science teaching, interdisciplinarity. 


\section{INTRODUÇÃO}

A necessidade de uma renovação no Ensino de Ciências já é muito discutida por diferentes autores (CACHAPUZ et all., 2005; KRASILCHIK, 2005; MARANDINO et al., 2009), talvez o que ainda não seja muito discutido dentro dessa renovação é a importância da integração entre as disciplinas científicas por meio de uma real interação destas.

Autores como Fazenda (2011), que discutem o tema interdisciplinaridade, atribuem a uma quase inexistente interação das disciplinas de Ciências. Para a autora, essa interação deve ir além de seus conceitos, sendo necessário permear, também, suas diretrizes, metodologias, procedimentos e organização de seu ensino. No entanto, a compreensão dessa terminologia se faz necessária para que seja possível trabalhar com as diferentes disciplinas científicas. De acordo com Japiassu (1976), existem terminologias muito próximas, mas de significados distintos. Contudo, os prefixos multi, inter, pluri ou trans, estarão sempre relacionados à palavra disciplina, que seria definida pelo autor como o conjunto específico de conhecimentos que tem suas características próprias no plano do ensino, da formação, dos métodos e dos materiais.

Sendo assim, para uma proposta mais didática, e, de acordo com as definições dadas por Japiassu (1976), destacam-se aqui alguns conceitos: Multidisciplinar, pode ser analisado como uma justaposição de duas ou mais disciplinas, com objetivos múltiplos, sem relação entre elas e nenhuma coordenação. Já a palavra Pluridisciplinar, deve ser definida como um conjunto de duas ou mais disciplinas, também com objetivos múltiplos, no entanto, com relações entre si, e, com certa cooperação, mas sem coordenação destas relações. O conceito de Interdisciplinar revela uma interação entre duas ou mais disciplinas, podendo ir da simples comunicação de ideias até a integração mútua dos conceitos, envolvendo epistemologia, terminologia, metodologia e organização da pesquisa, onde há coordenação desta integração. Por final, Transdisciplinar define-se como a coordenação de todas as disciplinas e interdisciplinas sobre a base de uma axiomática geral. Destina-se a um sistema de nível e objetivos múltiplos — há coordenação com vistas a uma finalidade comum.

Neste sentido, as pesquisas oriundas da área de Ensino de Ciências, mais especificamente, Ciências Biológicas podem estar relacionadas e integradas com diferentes disciplinas. Assim, para Japiassú (1976), a interdisciplinaridade ter-se-ia uma relação de reciprocidade, de mutualidade, ou melhor, um regime de copropriedade que iria possibilitar o diálogo entre os interessados, neste trabalho, o caso apresentado será uma proposta interdisciplinar envolvendo as disciplinas de Biologia e Estatística.

Já é descrito na literatura que o diálogo entre a Biologia e os métodos estatísticos, proporcionam um ótimo contexto para o ensino de Estatística (SEIER \& JOPLIN 2010).

Esse sentido de contextual corrobora com o sentido de integração, segundo Fazenda (2011), pode dizer-se que a interdisciplinaridade depende basicamente de uma atitude, aonde a colaboração entre as diversas disciplinas conduz a uma "interação", a uma intersubjetividade como única possibilidade de efetivação de um trabalho interdisciplinar.

Embora a pesquisa em biologia tenha sido a motivação para o desenvolvimento de vários métodos estatísticos, o ensino de estatística tem sido notoriamente difícil, em face das concepções equivocadas sobre o valor de dados estatísticos e o medo do tema, intrínseco ou aprendido (GARFIELD, 1995).

De acordo com a experiência dos autores, pode-se sugerir que, em grande parte, o distanciamento dos estudantes de biologia do conteúdo estatístico se dá pela forma que a disciplina muitas vezes é abordada, frequentemente desconectada das demais disciplinas: tal realidade faz com que os estudantes não reconheçam como os saberes estatísticos podem ser relacionados e aplicados em sua prática diária como biólogos. Possivelmente, tal fato pode ser agravado pela formação dos docentes que lecionam bioestatística, estes frequentemente graduados na área de exatas, podendo, assim, apresentar pouco domínio das ciências biológicas. Desta forma, não conse- 
guem contextualizar satisfatoriamente a ampla gama de utilidades dos saberes estatísticos, se restringindo aos exemplos dos livros-texto, usualmente característicos das ciências médicas.

Este quadro é evidenciado no levantamento realizado por A'Brook e Weyers (1995), no qual apresenta o ensino de estatística nos cursos de graduação em Ciências Biológicas do reino unido, sendo o mesmo quase sempre separado das disciplinas biológicas, e por esta razão os discentes não tem aprendido de fato estatística na graduação, o que leva a falta de tratamentos estatísticos, ou uso equivocado dos mesmos, por biólogos formados (HORGAN et al 1999).

A crescente preocupação com o ensino e aprendizagem da estatística em alguns países europeus e norte -americanos, ressaltada por Silva e colaboradores (2011), se deve, em grande parte, aos benefícios do desenvolvimento do raciocínio estatístico, tais como a melhor organização e análise de informações coletadas (WODEWOTZKI \& JACOBINI, 2005).

Desta forma, conforme Batanero (2001), Estrada e Batanero (2008) e Bazán e Aparício (2008) é evidenciado em suas pesquisas, nos saberes estatísticos, importantes ferramentas de tomada de decisão no dia a dia, como habilidades e conhecimentos estatísticos, que permitem ao indivíduo a reflexão e a formação de opiniões críticas (LOPES, 2002).

Neste contexto, a implementação do ensino de estatística deve acontecer de uma forma investigativa, conforme proposto por Mendonça e Lopes (2010), na qual o grupo de discentes vivencia a coleta e a análise de seus dados. Assim, baseado nesta perspectiva, a atividade prática descrita neste relato de experiência foi pautada.

\section{PÚBLICO-ALVO}

A disciplina "Ecologia e Educação Ambiental” é inserida no segundo período da matriz anual do curso de Ciências Biológicas de um centro universitário do interior do estado do Rio de Janeiro, nela os discentes são apresentados aos conceitos básicos de ecologia. A disciplina "Bioestatística", por sua vez, também faz parte deste segundo período da matriz anual do referido curso, nesta disciplina são abordados os conceitos mais basais de bioestatística. Já a disciplina "Diversidade Biológica e Zoologia dos Invertebrados" é apresentada a esses discentes no primeiro ano, nela os alunos estudam conceitos relativos à diversidade biológica e à sistemática e taxonomia de invertebrados.

O referido curso de Ciências Biológicas apresenta turmas com um perfil formado quase que exclusivamente por estudantes recém-saídos do ensino médio, sendo as turmas compostas usualmente por 30 alunos. A proveniência desses alunos pode ser do mesmo município do Centro Universitário, dos municípios do entorno no mesmo estado, ou, mesmo, de municípios vizinhos deste ou de outros estados, trazendo, assim, grande diversidade às turmas.

O Centro Universitário do referido curso é a maior instituição de ensino privado da região, localizado no município de Volta Redonda está situado às margens do Rio Paraíba do Sul, na Região do Médio Vale do Rio Paraíba, no eixo entre Rio de Janeiro e São Paulo, e abrange uma área de $181 \mathrm{~km}^{2}$, tendo sua origem se dado devido à instalação da Companhia Siderúrgica Nacional na década de 1940 (DIAS et al., 2004).

Como política pedagógica interna desta instituição de ensino, as avaliações bimestrais são realizadas por meio das chamadas Avaliações Diversificadas (AVDs) que, no caso do curso de Ciências Biológicas, curso anual, são de caráter somativo totalizando quatro conjunto de notas. Estas AVDs, ou conjunto de notas, não são constituídas apenas pelos testes habituais, mas, também, por outras formas de avaliação, tais como seminários, trabalhos ou relatórios.

A atividade a qual este relato de experiência se refere constituiu uma avaliação formativa, correspondente a 40\% da nota da terceira AVD das disciplinas Ecologia e Educação Ambiental e Bioestatística. 


\section{DESCRIÇÃO DAATIVIDADE}

Em um primeiro momento, os alunos foram conduzidos até o jardim do campus, no qual estão presentes várias espécies arbóreas e arbustivas, onde após um exercício de observação da turma, uma série de perguntas foi levantada pelo professor, tais como: 1 ) Como eles achavam de deveria ser a distribuição das formigas por aquelas plantas? 2 ) Haveria diferença na composição de espécies de formigas associadas de uma espécie de planta para a outra? 3 ) Haveria diferença na composição de comunidades de formigas em diferentes alturas da mesma árvore? 4 ) Haveria diferença na composição da fauna de formigas de árvores mais próximas à estrada, em relação às presentes mais no interior do campus?

Este exercício coletivo os levou, a partir de seus conhecimentos de ecologia, a formularem algumas hipóteses, entre elas, que "a distribuição não poderia ser homogênea, pois as espécies de formigas teriam diferentes nichos, e, ocupariam diferentes microhabitats!"

Assim, um exercício conjunto de construção de hipóteses se seguiu, fazendo com que aqueles conceitos que haviam sido expostos em sala de aula ganhassem forma, a partir de um exemplo bem próximo, com o qual eles poderiam facilmente se relacionar.

Passada esta etapa do levantamento de hipóteses, os alunos eram questionados sobre como poderiam testá-las. Este questionamento se mostra fundamental para a inserção dos conceitos de estatística nesta questão basicamente ecológica, visto que, seria necessário construir um experimento que pudesse testar cientificamente aquelas hipóteses que haviam acabado de ser formuladas.

Neste segundo momento, a turma foi reconduzida à sala de aula, onde o exercício de construção coletiva do desenho experimental teria início. Nesta etapa, cada pergunta e cada hipótese seriam analisadas conjuntamente pela turma, para que o experimento fosse confeccionado com a contribuição de todos.

Consequentemente, não foi difícil surgir, por parte dos discentes, diversos questionamentos como o tipo de amostra deveria ser utilizada, ou, com quantas réplicas deveriam trabalhar.

A partir desses questionamentos, foi realizada a construção conjunta do experimento, que demandava o entendimento dos conceitos de ecologia e de bioestatística simultaneamente. Exemplificando essa interdisciplinaridade, para que fosse possível aos discentes a verificação de semelhanças ou diferenças de organismos nas árvores mais próximas à estrada ou nas mais distantes, era essencial que se explorasse um gradiente ambiental. Quando os alunos concluíam a necessidade de execução desta etapa, era questionado aos mesmos qual seria a melhor forma de se executar isso, e, qual seria o tipo de amostragem que deveria se aplicar nesses casos. Por exemplo, seria uma amostragem sistemática, ou não? Aos poucos, os conceitos destas duas disciplinas dialogavam entre si.

Posteriormente a essa fase, a turma foi informada que o professor de Diversidade Biológica e Zoologia dos Invertebrados os receberiam no laboratório didático de Zoologia, assim, os alunos juntamente a este docente poderiam proceder para a identificação do material coletado, além de auxiliar na coleta. A partir do momento que todo o desenho do experimento ficou pronto, e que todos, discentes e docentes estavam cientes de sua função, pode ser realizada a atividade de coleta. Esta aconteceu no próprio campus, como já descrito anteriormente, algumas horas antes das aulas de Ecologia e Educação Ambiental e Bioestatística, para que a mesma pudesse ocorrer com a luz do dia, já que as aulas são noturnas. A turma então foi dividida em quatro grupos de seis alunos, para cada grupo coube amostrar uma determinada área limitada, uma faixa de observação, chamada de transecto, composto de três diferentes espécies arbóreas, posicionado em paralelo à estrada que passa em frente ao Campus.

Os transectos foram posicionados distando aproximadamente 10 metros um em relação ao outro, de forma que fosse observado um pequeno gradiente em relação à distância da estrada: cada grupo deveria se responsabilizar pelo seu material coletado, e sua posterior identificação, no laboratório didático de zoologia. 
Com o conjunto de dados de cada transecto, os alunos, através das análises estatísticas apropriadas, seriam capazes de responder às questões 1,2 e 3 . Devendo com essas respostas redigir um relatório de pesquisa, no qual os procedimentos estatísticos necessitariam ser devidamente apresentados e justificados, além disso, as hipóteses seriam explicadas à luz da teoria ecológica.

Entretanto, para a última pergunta "Haveria diferença na composição da fauna de formigas de árvores mais próximas à estrada, em relação às presentes mais no interior do campus?”, se faria necessário algo mais: os grupos deveriam combinar suas respectivas planilhas de forma a gerar uma matriz de dados maior, que precisava ser analisada para que as hipóteses referentes à pergunta pudessem ser testadas.

A continuidade deste trabalho seria mais interdisciplinar ainda, visto que, uma vez estabelecido o desenho experimental, a participação de outra disciplina seria fundamental, afinal, as formigas coletadas deveriam ser identificadas, através dos conceitos fornecidos na disciplina Diversidade Biológica e Zoologia dos Invertebrados.

Após a amostragem os discentes se dirigiram ao laboratório para que cada grupo pudesse triar, montar, rotular e identificar os espécimes coletados. No laboratório, com auxílio de chaves dicotômicas de Bolton (1994) os discentes identificaram as subfamílias neotropicais e os gêneros. As espécies foram identificadas com auxílio de chaves contidas em revisões taxonômicas disponíveis.

Uma vez coletados e identificados os dados, a turma foi novamente reunida em sala para que os passos seguintes fossem discutidos. A estrutura das planilhas com as quais eles trabalhariam foram, então, construídas de forma coletiva.

A partir disso, foi estabelecido que cada grupo apresentaria seus resultados sob a forma de um relatório de pesquisa, seguindo o formato de um manuscrito científico, no qual eles deveriam apresentar seu objeto de estudo, suas hipóteses, metodologia, e fundamentar alicerçados na teoria ecológica as suas conclusões, com base nos padrões evidenciados pelas análises estatísticas para seus dados.

Nas apresentações dos grupos, o que se pôde observar foram diferentes soluções metodológicas para responder às perguntas propostas. Enquanto alguns grupos optaram por testes de hipóteses simples, outros optaram por trabalhar com índices de similaridade e diversidade, proporcionando, desta forma, diferentes abordagens para o mesmo tema.

As apresentações foram também permeadas por informações da ecologia e bionomia das espécies encontradas, levantadas pelos alunos a partir de levantamento bibliográfico, as quais eram confrontadas pelos grupos com os resultados de seus respectivos testes de hipóteses e/ou análises de similaridade.

Foi possível observar uma construção de raciocínio e desenvolvimento de maturidade em termos de pesquisa de acordo com as respostas apresentadas no manuscrito. Essa observação se deu desde as respostas mais simples, quando os trabalhos de análise eram restritos ao grupo de seis alunos e seu respectivo transecto, até o momento da análise comparativa das diferentes faixas de observação em relação à proximidade com a estrada.

Desta forma ocorreu um perfeito diálogo entre as disciplinas envolvidas neste estudo, o qual foi exaltado pelos discentes, que afirmaram que mais atividades integradoras como essa deveriam ser realizadas em seu curso.

\section{CONCLUSÃO}

No ensino de ciências o fato do aluno conviver e interagir com fenômenos que são objetos de estudo dessas ciências para além dos muros das escolas desautoriza a suposição de que uma compreensão deles seja obtida apenas por sua abordagem em sala de aula. 
Diversas práticas como trabalhos de campo, aulas em espaços não formais, aulas práticas em laboratórios ou ao ar livre, fazem com que os aprendizes participem da construção de uma pedagogia contextualizada, além disso, constroem juntamente com os docentes seu próprio conhecimento.

Não se pode deixar de levantar que essa construção do conhecimento se fez à luz de significados, reais, vivenciados e observados pelo próprio aluno que participou dessa aula prática. $\mathrm{O}$ aluno, imbuído de conhecimento prévio sobre aquele habitat no qual ele frequenta por dias durante os anos cursados nesta graduação, tem a oportunidade de somar novos conhecimentos, interdisciplinares, desta vez conduzidos pela cientificidade, e, com significado, levando-os a um real aprendizado.

Para esse relato pode-se observar que o mínimo alcançado foi o prazer dos discentes em realizar uma prática palpável, em um ambiente familiar, que pudesse unir saberes de diferentes disciplinas. E, o máximo foi a união dessas disciplinas em um enfoque interdisciplinar.

\section{REFERÊNCIAS}

A'Brook R., Weyers J.D.B. Teaching of statistics to UK undergraduate biology students in 1995. J. Biol. Educ. 1996, 30:281-288.

BAZÁN, J.L.; APARICIO, A. Una revisión de la estadística y la probabilidad en la estructura curricular peruana. ArIsTAS. Revista de los educadores del Colegio Bertolt Brecht, 2008.

CAHAPUZ, A.; GIL-PEREZ, D.; CARVALHO, A. M. P.; PRAIA, J. E VILCHES, A. (orgs.) - A necessária renovação do ensino de Ciências. São Paulo: Cortez, 2005.

ESTRADA, A.; BARANERO, C. Explaining teachers' attitudes towards statistics._In C. Batanero, G.., Burrill, C., Reading. y A. Rossman, A.,. Joint ICMI/IASE Study: Teaching Statistics in School Mathematics. Challenges for Teaching and Teacher Education. Proceedings of the ICMI Study 18 and IASE 2008, Round Table Conference. Monterrey, Mexico, International Commission on Mathematical Instruction and International Association for Statistical Education, 2008.

FAZENDA, Ivani; C. Arantes. Integração e Interdisciplinaridade no Ensino Brasileiro: Efetividade ou Ideologia. 6. Ed.: São Paulo: Edições Loyola, 2011.

HORGANG. W., ELSTON D. A., FRANKLiN M. F., GLASBEY C. A., HUNTER E. A., TALBOLT M., KEMPTON R. A., McNICOL J. W., WRIGHTF. Teaching statistics to biological research scientists. Statistician. 1999, 48:393-400.

JAPIASSU, Hilton. Interdisciplinaridade e Patologia do Saber. Rio de Janeiro: Imago, 1976.

KRASILCHICK, Myriam. Prática de Ensino de Biologia. 4 ed. rev. e ampl. $1^{\text {a }}$ reimpr. São Paulo: Editora da Universidade de São Paulo, 2005.

MARANDINO, M.; SELLES, S. E.; FERREIRA, M. S. Ensino de Biologia: histórias e práticas em diferentes espaços educativos. São Paulo: Cortez, 2009.

SEIER, E.; JOPLIN, K.H. 2010. C. READING (Ed.). Data and context in statistics education: Towards an evidence-based society. Proceedings of the Eighth International Conference on Teaching Statistics (ICOTS8, July, 2010), Ljubljana, Slovenia. Voorburg, The Netherlands: International Statistical Institute. www.stat. auckland.ac.nz/ iase/publications.php 